und eine neue der Anissäure homologe Säure.

und dessen Zusammenselzung sich der Formel $\mathrm{E}_{9} \mathrm{H}_{9} \mathrm{Ag} \theta_{3}$ ganz entsprechend ergab.

\title{
Bestimmung der zur Verbrennung organischer Stoffe nöthigen Sauerstoffmenge;
}

von August Stromeyer.

Der Sauerstoffgehalt einer organischen Substanz lärst sich nach v. Baumhauer ${ }^{*}$ ) direct bestimmen, indem man die Elementaranalyse mit Kupferoxyd in einer an beiden Enden offenen Röhre vornimmt, welche mit graduirten Cylindern in Verbindung stehen. Der eine derselben enthält Sauerstoffgas, welches nach Beendigung der Verbrennung hindurchgeleitet wird, um das entstandene metallische Kupfer wieder zu oxydiren. Der Verbrauch an Sauerstoff, welcher an beiden Cylindern gemessen wird, abgezogen von dem Sauerstoffgehalt der aufgefangenen Kohlensäure und des Wassers, giebt den der organischen Substanz. Versuche mil Kleesäure und kleesaurem Bleioxyd gaben auf diese Weise sehr genaue Resultate.

Schon Prout**) hat den Vorschlag gemacht, man solle, um den vom Kupferoxyd abgegebenen Sauerstoff zu bestimmen, den Rückstand in der Röhre mit verdünnter Schwefelsäure behandeln, welche eine der Menge des Sauerstoffs

*) Diese Annalen XC, 230.

**) Berzelius, Lehrbuch der Chemie, Bd. III, Abtheilung 1, S. 167 (1827). 
entsprechende Quantität metallischen Kupfers ungelöst zurückläIst.

Ich habe, diesen Vorschlag verfolgend, das Kupfer nach einem von Fleitmann*) angegebenen Verfahren durch Auflösen des Rückstandes der Verbrennung in Salzsäure und Eisenchlorid und Titrirung des entstandenen Eisenchlorürs mit Chamäleon bestimmt :

$$
\mathrm{Cu}+\mathrm{Fe}^{2} \mathrm{Cl}^{3}=\mathrm{CuCl}+2 \mathrm{FeCl} \text {. }
$$

Es war zu diesem Zwecke erforderlich, dals das anzuwendende Kupferoxyd kein Oxydul enthalte, welches bei der Darstellung durch Ausglühen des salpetersauren Oxyds in Tiegeln fast unvermeidlich sich durch die Ofengase bildet. Ich habe es mir defshalb aus kohlensaurem Kupferoxyd $\left(2 \mathrm{CuO}, \mathrm{CO}^{2}+\mathrm{HO}\right)$ durch mälsiges Erhitzen in einem Glaskolben dargestellt. Letzteres war durch kochende Fällung von Kupfervitriol mit etwas mehr als seinem Aequivalent kohlensauren Natrons bereitet. Man kann dazu ohne Nachtheil einen kupfernen Kessel anwenden, mufs nur die Lösungen der Salze vorher filtriren, da sie oft Holzspänchen enthalten, so wie Beimengung von Papierfasern sorgfältig verhüten. Dann ist das daraus erhaltene Kupferoxyd aber auch ganz frei von $0 x y d u l$, löst sich vollständig in verdünnter Schwefelsäure, und die Lösung in Salzsäure gieb mit keinem der von $\mathrm{Schiff*}$ ) angegebenen Reagentien z. B. molybdänsaurem Ammoniak, eine Anzeige darauf.

Das kohlensaure Kupferoxyd selbst zur Verbrennung anzuwenden, ist nicht zu empfehlen, da wegen der starken Gasentwickelung leicht ein Herausschleudern aus der Röhre stattfindet. Auch ist es noch voluminöser wie das daraus erhaltene 0xyd, und dieses ist schon so leicht, dafs es einen

*) Diese Annalen XCVI, 215.

**) Daselbst CXII, 372. 
dreimal gröfseren Raum einnimmt, wie das durch Glühen des salpetersauren Salzes erhaltene. Es verbrennt organische Substanzen mit grolser Leichtigkeit, aber die Kohlensäure entwickelt sich zu rasch zur vollständigen Absorption im Kaliapparat, wefshalb auch Liebig *) seinen Gebrauch zur Elementaranalyse widerräth. Aber zur Bestimmung der zur Verbrennung nöthigen Sauerstoffmenge eignet es sich gerade ganz vorzüglich, wenn man dabei auf die Auffangung des Wassers und der Kohlensäure verzichtet. Da es so ausnehmend reducibel ist, gebraucht man viel weniger davon, als sonst bei einer Elementaranalyse angewendel wird. Organische Stoffe, welche so viel oder mehr Sauerstoff enthalten, um mit dem Wasserstoff Wasser zu bilden, erfordern zu der Mengung etwa dreimal so viel desselben, wie der Berechnung nach nothwendig sein sollte, und die, welche überschüssigen Wasserstoff enthalten, viermal so viel. Ich habe dann der Sicherheit wegen allerdings noch eben so viel davor gegeben; indefs erstreckt sich das reducirte Kupfer, aufser bei flüchtigen Substanzen, wenig über das Gemenge hinaus.

Dem Kupferoxyde menge ich die Hälfte seines Gewichts von trockenem kohlensaurem Natron zu. Dieses Gemenge sintert beim Glühen und verbrennt dadurch die letzten Antheile der Kohle. Selbst schwer verbrennliche Substanzen, wie Steinkohle, werden dadurch ganz vollständig verbrannt, so dafs beim Auflösen in Eisenchlorid keine Kohle zurückbleibt.

Der Schwefel in organischen Stoffen verbrennt damil vollständig zu schwefelsaurem Natron. - Das Chlor bildel Chlornatrium, wobei nicht zu vergessen ist, dafs dadurch der Sauerstoff des Natrons ausgetrieben und zur Bildung

*) Handwörterbuch der Chemie, Bd. I, 374. 
von Kohlensäure und Wasser verwendet wird. Das Chlor ist also hierbei wie Sauerstoff $2 u$ rechnen.

Auf die Anwendbarkeit dieses Verfahrens zu Stickstoff enthaltenden organischen Körpern hatte ich Anfangs Verzicht geleistet, da der Gebrauch von vorgelegtem metallischem Kupfer, um das hierbei entstehende Stickstoffoxyd zu desoxydiren, hier natürlich ausgeschlossen war. Es scheint indels, als müsse das kohlensaure Natron die Entslehung desselben verhindern, denn ich erhielt bei den Versuchen mit mehreren Stickstoffkörpern sehr nahe den berechneten Sauerstoffverbrauch *).

Nur die Nitroverbindungen und die salpetersauren organischen Basen, von welchen ich freilich blofs den salpetersauren Harnstoff versucht habe, ergaben einen bedeutend zu grofsen Verbrauch, so dals hier Stickstoffoxyd oder Oxydul entwichen sein mufs.

Das aus kohlensaurem Kupferoxyd bereitete $0 x y d$ läfst sich mit der organischen Substanz und dem kohlensauren Natron nicht gut in einem Mörser verreiben, weil es zu stark an demselben haftet; es reicht indessen hin, sie in einem glatten Schälchen mit einem kleinen Löffel gut $\mathrm{zu}$ vermengen. Man füllt sie dann mittelst eines Trichterchens in eine Glasröhre und giebt noch eben so viel Kupferoxyd davor. Das lelztere habe ich gut gefunden wie Schielspulver zu körnen. Es wird mit $1 / 10$ kohlensaurem Natron und Wasser $\mathrm{zu}$ einer passenden Consistenz angemacht und durch ein Blechsieb mit Löchèrn von einer Linie Durchmesser gerieben, dann getrocknet und der feine Slaub davon abgesiebt.

*) Es möchte danach zu empfehlen sein, bei der directen Bestimmung des Stickstoffs als Gas dem Kupferoxyde kohlensaures Natron zuzusetzen. 
Die Glasröhre verbindet man miltelst eines Korkes oder bei kleinem Durchmesser durch eine Caoutchoucröhre mit einem fein ausgezogenen Glasröhrchen. - Durch Aufklopfen auf einen Tisch stellt man einen Canal über dem Gemenge her. Sie wird dann in dem zur Elementaranalyse dienenden Ofen, von vorn nach hinten fortschreitend, in kleinen Stücken zum Glühen erhitzt.

Man lege sie dazu in eine aus Eisenblech gebogene, mit Pulver von Holzkohle oder besser Coaks gefüllte Rinne, wodurch man vor dem Zerspringen und Zusammenschmelzen besser gesichert ist. Nachdem die ganze Röhre glüht, schmilzt man die Oeffnung des Glasröhrchens mit dem Löthrohre zu und läfst erkalten. Um letzteres zu beschleunigen, kanni man die Kohlen wegräumen und die Rinne mit der Röhre aus dem Ofen ziehen.

Hat man gute Glasröhren und ist die Hitze nicht zu stark gewesen, so kann man den Inhalt fast ganz in eine Digerirflasche ausschütten und das wenige anhängende in der Röhre selbst auflösen. Ist sie aber zusammengefallen oder von engem Durchmesser, so ist es am bequemsten, sie über einem Bogen Glanzpapier in Stücke zu zerbrechen, welche sich in die Flasche bringen lassen. Es läfst sich diefs ohne Verlust ausführen, wenn man die Röhre Stellenweise über einer kleinen Spirituslampe erhitzt und dann mit einem nassen Haarpinsel einen Strich herummacht. Sie erhält dann Sprünge an dieser Stelle und läfst sich nun ohne Gewalt zerbrechen, so dafs nichts umherspringt.

Ich habe auch die ganze Röhre, nachdem ich die Spitze abgebrochen hatte, in eine weitere Glasröhre gesteckt und darin mit dem Auflösungsmittel erhitzt; indefs ist das Kochen schwierig darin, und das Zerbrechen gelingt recht gut.

Zu meinen ersten Versuchen habe ich nach F leitmann's Vorschrift Eisenchlorid und Salzsäure angewendet, nachher 
aber dieselben gegen schwefelsaures Eisenoxyd $\left(\mathrm{Fe}^{2} \mathrm{O}^{3}, 3 \mathrm{SO}^{3}\right)$ und verdünnte Schwefelsäure vertauscht, weil das hier entstehende schwefelsaure Eisenoxydul viel weniger empfindlich gegen den Sauerstoff der Luft, wie das Eisenchlorür ist. Bei dem letzleren mufste man sich mit der Titrirung beeilen, während eine mit schwefelsaurem Eisenoxyd erhaltene Lösung, welche verdiunt in einer offenen Flasche gestanden hatte, sich noch am anderen Tage ganz unverändert zeigte. - Auch war es nicht erforderlich, das Wasser zur Verdünnung vorher durch Kochen von Luft zu befreien.

Man könnte die Eisenlösung ganz entbehren und den Inhalt der Röhre blofs in Salzsäure lösen, da genug Kupferoxyd noch vorhanden ist, um alles melallische Kupfer in Chlorür zu verwandeln. Durch Zusatz von Kochsalz kann man letzteres bei der nöthigen Verdünnung aufgelöst erhalten. Allein diefs ist durchaus nicht zu empfehlen, weil die Kupferchlorürlösung gar zu schnell sich an der Luft oxydirt.

Ich habe eine Auflösung mit etwa 8 pc. Eisenoxyd von passender Concentration gefunden. Da man aus der Elementaranalyse schon die zur Verbrennung nöthige Sauerstoffmenge kennt, welche diese directe Bestimmung nur controliren soll, so kann man die zur Oxydation des entstandenen Kupfers nöthige Menge von Eisenoxyd berechnen. 1 Alom $\mathrm{Fe}^{2} 0^{3}$ (80) giebt 1 Atom $O$ (8) an 1 Atom $\mathrm{Cu}$ ab. Man bedürte also zehnmal so viel $\mathrm{Fe}^{2} \mathrm{O}^{3}$, wie das Kupferoxyd an Sauerstoff abgegeben hat. Dabei würde jedoch die Auflösung des Kupfers zu träge vor sich gehen. Man verdoppelt defshalb die gefundene Menge, wobei die Lösung rasch von Stalten geht. Der Eisenoxydlösung, welche natürlich ganz frei von 0xydul, so wie von Salpetersäure sein mufs, selat man etwas mehr destillirte, oder wenigstens von Stoffen, welche das Chamäleon reduciren, freie Schwefelsäure $\mathrm{zu}$, wie 
das angewandte Kupferoxyd und kohlensaure Natron erfordern. Die Digerirflasche verschliefst man mil Mohr's*) Caoutchoucventil und erhitzt sie auf einem kleinen Sandbade über der Spirituslampe, bis alles Kupfer gelöst ist, wozu man eine Zeit lang kochen lassen mufs. Wenn die Hitze bei der Verbrennung nicht zu stark gewesen ist, nimmt dieses Lösungsmillel Alles auf, so dafs die Stücke der Glasröhre ganz entfärbt werden. Bei zu starker Hitze bilden sich aber rothe Flecke am Glase, vermuthlich ein Kupferoxydulsilicat, welche der Einwirkung desselben widerstehen. Diese lösen sich nun in Eisenchlorid und Salzsäure. Nach dem Erkalten giefst man daher die schwefelsaure Lösung in eine Mafsflasche, spült mit Wasser nach, sucht die etwa roth gefärbten Stellen der Röhre aus und kocht sie in einer kleineren Flasche mit Salzsäure und Eisenchlorid. Die erhaltene, stets nur sehr wenig betragende Lösung vereinigt man mit der schwefelsauren, bringt durch Wasserzusatz auf ein bestimmtes $\mathrm{V}_{0-}$ lumen, schültelt sorgfältig durch und titrirt nun davon abgemessene Antheile, z. B. 1/4 der Lösung, mit Chamäleon. Die verdünnte Lösung mufs die Farbe des Kupfervitriols haben; ist sie gelbgrün, so fehlt es an Schwefelsäure, wovon man dann noch etwas zusetzt.

Eine Auflösung von Kupferoxyd, wenn auch ganz frei von Oxydul, erfordert mehr Chamäleon zur Röthung, wie ein gleiches Volumen Wasser, weil die blaue Farbe der Lösung erst durch die rothe des Chamäleons aufgehoben wird, so dafs sie fast ganz wasserhell erscheint. - Auch das schwefelsaure Eisenoxyd vermehrt etwas den Verbrauch an Chamäleon. Um den daraus entstehenden Fehler za verbessern, löst man von dem zur Verbrennung benutzten Kupferoxyd - reinem und gekörntem - so viel, wie in dem

*) Lehrbuch der Titrirmethode Bd. I, 144. 
zur Titrirung benutzten Antheile der Lösung enthalten ist, in verdünnter Schwefelsäure auf, setzt auch den darauf kommenden Antheil schwefelsaures Eisenoxyd zu, verdünnt eben so stark wie vorher, und setzt nun von demselhen, aber hierzu zweckmälsig zehnfach verdünnten, Chamäleon bis zur Entfärbung und Röthung zu. Die verbrauchte Menge Chamäleon wird dann von der vorhin erhaltenen abgezogen.

Bei der Titrirung verdünne man so stark, dals auf 100 cc. nur etwa $0,3 \mathrm{Grm}$. Kupferoxyd kommen, weil bei mehr die Flüssigkeil durch das Chamäleon nicht ganz entfärbt wird und defshalb die Röthung nicht so deullich wahrzunehmen ist. Die letztere verschwindet bald wieder, aber die Farblosigkeit bleibt eine Zeit lang, so dafs man über den Punkt der vollendeten Oxydation nicht unsicher sein kann.

$0,103 \mathrm{Grm}$. Rohrzucker wurden mit 1,5 Kupferoxyd und 0,75 kohlensaurem Natron gemengt und noch 1,5 $\mathrm{CuO}$ vorgelegt. Nach dem Glühen wurden sie in $30 \mathrm{CC}$. einer Auflösung von schwefelsaurem Eisenoxyd za $8 \mathrm{pC}$. $\mathrm{Fe}^{2} 0^{3}$ und 5 CC. destillirtem Vitriolöl gelöst and zu $250 \mathrm{CC}$. verdünnt. Davon wurden je 50 CC. zu 200 CC. verdünnt. Sie erforderten bei dreimaliger Wiederholung jedesmal genau 24,4 CC. Chamäleon. Es wurden nun 0,6 des angewandten Kupferoxyds in verdünnter Schwefelsäure gelöst, $6 \mathrm{CC}$. der Eisenoxydlösung zugeselzt und ebenfalls zu $200 \mathrm{CC}$. verdünntSie erforderten 0,5 CC. des Chamäleons zur deutlichen Röthung. Diese müssen von $\mathbf{2 4 , 4}$ abgezogen werden, bleiben also 23,9 oder fürs Ganze 119,5. - 22,5 des Chamäleons entsprechen 0,151 Eisen in verdünnter Schwefelsäure gelöst, also $119,5=0,80197$ Eisen oder 0,114567 Sauersloff. Danach würde 1 Atom Rohrzucker $\left(\mathrm{C}_{12} \mathrm{H}_{11} \mathrm{O}_{11}\right)$, Atomgewicht $=$ 171, zur Verbrennung 190,2 Sauerstoff verbraucht haben, während die Theorie 192 (24 Atome 0) verlangt. 
Die Titrirungen gleicher Antheile stimmten fast immer genau auf $0,1 \mathrm{CC}$. überein; angenommen indefs, die Unsicherheit betrüge 0,1 oder hier 0,5 fürs Ganze, so wären das $0,00335 \mathrm{Fe}$ oder $0,000478 \mathrm{o}$, was für 1 Atom Zucker (171) $0,79 \sigma$ oder etwa $1 / 10$ Atom ausmacht. Leider geben nun fast alle meine Versuche, wie dieser, weniger Sauerstoffverbrauch wie die Theorie erfordert. Vielleicht ist die atmosphärische Luft in der Röhre die Hauptursache dieses Verlustes. Bei diesem Versuche hatle die angewendete Glasröhre einen Inhalt von $10 \mathrm{CC}$. Berechnet man den Raum, welchen Kupferoxyd und kohlensaures Natron einnahmen, nach ihrem spec. Gewichte und reducirt für Thermometer- und Baromelerstand auf 0 und $760^{\mathrm{mm}}$, so vermindern die $10 \mathrm{CC}$. sich auf 8,75. Davon 20,9 pC. $0=1,8 \mathrm{CC}$. Da aber die Erwärmung allmälig ist, werden bis $100^{\circ}$, wo die organische Substanz noch unverändert ist, diese auf $1,3 \mathrm{CC}$. abnehmen. Das sind immer noch 1,8 Milligrm. Sauerstoff, und bei obigem Versuch mit Zucker beträgt der Verlust nur circa 1 Milligrm. Ich habe versucht, die atmosphärische Luft dadurch auszutreiben, dafs ich doppelt-kohlensaures Natron ans Ende der Röhre brachte und zuerst erhitzte. Es gewährte diefs einigen aber nur geringen Nutzen. Hätte ich eine Luftpumpe zur Disposition gehabt, so würde ich Liebig's*) Apparat der Stickstoffbestimmung dazu versucht haben, bei welchem die Röhre mittelst eines dreischenkeligen Stückes mit einer 30 Zoll langen unter Quecksilber tauchenden Gasentbindungsröhre und mit der Luftpumpe verbunden wird. Durch Auspumpen und abwechselndes Füllen mit Kohlensäure, welche aus am Ende der Röhre angebrachtem kohlensaurem Kupferoxyd durch Erhitzen entwickelt wird, kann die Röhre gänzlich von atmosphärischer Luft befreit werden. Vielleicht

*) Handwörterbuch der Chemie Bd. I, 386. 
gelingt es damit, sich dem theoretischen Verbrauch mehr zu nähern, als es mir möglich war. Bis jelzt ist der Verlust, wiewohl an sich nicht grols, doch noch zu hoch, um daraus den procentischen Sauerstoffgehalt der organischen Substanz nach v. Baumhauer zu berechnen, indem man den Verbrauch vom Sauerstoffgehalt der Kohlensäure und des Wassers, welche die Elementaranalyse geliefert hat, abzieht. Die Zahlen fallen zu hoch aus und können die Bestimmung aus dem Verlust nicht corrigiren. In der That ist dazu eine sehr genaue Bestimmung des Sauerstoffverbrauchs nothwendig, denn da man bei der Analyse nur wenig Kohlensäure verliert und vom Wasser gewöhnlich etwas zu viel erhält, muls ein minus im Verbrauch den Sauerstoffgehalt der organischen Substanz zu hoch ausfallen machen. Selbst v. Baum hau er's sehr genauer Versuch mit Kleesäure giebt denselben ein wenig zu hoch und die Analyse einen kleinen Ueberschufs.

Da jedoch der Verlust bei meinen Versuchen sich meistens nur auf $1 / 4$ Atom und weniger Sauerstoff berechnet, möchte ich doch glauben, dafs diese Probe eine nützliche Controle der durch Elementaranalyse und Atomgewichtsbestimmung gefundenen Resultate abgeben könne.

Da sie sehr leicht und schnell auszuführen ist, möchte man sich ihrer auch in vielen Fällen zur Bestimmung schon bekannter Körper bedienen können, bei welchen man jetzt wegen Mangel entscheidender Reactionen zur Elementaranalyse genöthigt ist.

Auch wird sie zur Bestimmung des Wärmeffectes der Brennmaterialien anwendbar sein anstatt Berthier's Probe mit Bleioxyd, bei welcher nach dessen eigenen Versuchen *) ein Entweichen brenzlicher Producte nicht zu vermeiden ist.

*) Berthier, Traité des essais r, 378, z. B. Versuche mit Kleesäure, Weinsäure, Gummi u. s. w. 
Nachdem neuere Untersuchungen die Unrichtigkeit von W elter's Gesetz erwiesen haben, und da ein gleiches Gewicht Sauerstoff, wenn es sich mit Wasserstoff zu Wasser verbindet, doppelt so viel Wärme entwickelt, wie mit Kohle zu Kohlensäure, kann freilich nur die Elementaranalyse genaue Zahlen liefern. Berthier's Probe wird indessen noch häufig angewendet, und die mit Kupferoxyd, welche nicht mehr Zeit und Kosten erfordert, würde doch genauere Resultate liefern. Ich habe bei einer Steinkohle, deren Zusammenselzung ich durch die Elementaranalyse bestimmt hatte, den danach berechneten Sauerstoffverbrauch sehr nahe durch diese Probe erhalten.

Die von mir mit einigen organischen Stoffen erhaltenen Resultate sind nun folgende :

Rohrzucker $\mathrm{C}^{12} \mathrm{H}^{11} \mathrm{O}^{11}$. Atom 171 erfordert 24 At. $\mathrm{O}$.

$0,103 \mathrm{Grm}$. in dem oben angeführten Versuch gaben 190,2 O statt.192.

$0,202 \mathrm{Grm}$. mit $3 \mathrm{Grm}$. $\mathrm{CuO}$ und 1,5 $\mathrm{NaO}, \mathrm{CO}^{2}$ gemengt und noch 3,0 gekörntes $\mathrm{CuO}$ vorgelegt. - In $50 \mathrm{CC}$. Löstung von schwefelsaurem Eisenoxyd à $8 \mathrm{pC}$. $\mathrm{Fe}^{2}()^{3}$ und $8 \mathrm{CC}$. destillirtem Vitriolöl gelöst und zu 1 Liter verdünnt. $250 \mathrm{CC}$. davon zu 500 verdünnt erforderten in zwei Versuchen 48,6 eines Chamäleons, von welchem 17,3 CC. $=1$ Grm. schwefelsauren Fisenoxydul - Ammoniak oder 0,020408 Sauerstoff waren. Es wurden nun $0,75 \mathrm{CuO}$ und 0,75 gekörntes $\mathrm{CuO}$ in verdünnter Schwefelsäure gelöst, 12,5 CC. der Eisenoxydlösung zugesetzt und gleichfalls zu 500 CC. verdünnt. Sie erforderten $0,9 \mathrm{CC}$. Chamäleon zur Röthung, welche also von 48,6 abzuziehen sind $=47,7$. Diese mit 4 multiplicirt geben 190,8 fürs Ganze $=0,2250710$. Das macht für 1 Atom Rohrzucker 190,5 O statt 192 (24 At.), also fast dasselbe Resultat, wie vorher.

Es ist nun wohl überflüssig, bei den anderen Versuchen wieder das jedesmalige Detail der Titrirungen anzuführen. Ich will nur die angewendeten Mengen der organischen Substanz und des damit gemengten Kupferoxydes angeben und für alle bemerken, dafs dem letzteren stets die Hälfte seines 
Gewichts von kohlensaurem Natron beigemengt war, dann aber noch eben so viel Kupferoxyd, entweder allein oder mit $1 / 10$ kohlensaurem Natron gekörnt, vorgelegt wurde.

Wo Eisenchlorid und Salzsäure angewendet wurden, habe ich es angemerkt, sonst ist schwefelsaures Eisenoxyd gebraucht.

Den Titer des Chamäleons habe ich nit Eisen in verdünnter Schwefelsäure gelöst oder mit dem schwefelsauren Eisenoxydul-Ammoniak genommen. Letzteres, worüber ich auf Mohr's Lehrbuch der Titrirmethode*) verweise, ist bequemer, es muls aber richtig getrocknet sein. Etwas gröfsere Krystallbrocken schliefsen leicht noch Mutterlauge ein. Es mul's nahezu dasselbe Resultat geben, wie die darin enthaltene Menge Eisen $(1 / 7)$ giebt. - Den Titer des Chamäleons habe ich täglich genommen, er verändert sich übrigens sehr langsam.

Milchzucker $\mathrm{C}^{24} \mathrm{H}^{22} \mathrm{O}^{22}+2$ aq. Atom 360 erfordert 48 At. $\mathrm{O}$.

$0,113 \mathrm{Grm}$. mit 1,5 CuO gaben 0,12013 O. 1 At. also 382,7 statt 384 .

Kleesäure $\mathrm{C}^{2} \mathrm{H}^{3} \mathrm{O}^{6}$. Atom 63 erfordert $1 \mathrm{At}$. 0 .

$0,63 \mathrm{Grm}$. mit $\mathbf{1 , 5} \mathrm{CuO}$ gaben $0,0797 \mathrm{O}$ statt 0,08 .

Weinstein $\mathrm{C}^{8} \mathrm{H}^{5} \mathrm{KO}^{12}$. Atom 188,2 erfordert 10 At. $\mathrm{O}$.

$0,202 \mathrm{Grm}$. mit 1,5 CuO gaben 0,084797 0. 1 Atom also 78,92 statt 80 .

Bleizucker $\mathrm{C}^{4} \mathrm{H}^{3} \mathrm{PbO}^{4}+3$ aq. Atom 190 erfordert 8 At. 0 .

$0,303 \mathrm{Grm}$. mit $1,5 \mathrm{CuO}$, mit $\mathrm{Ee}^{2} \mathrm{Cl}^{3}$ gaben 0,100218 o. 1 Atom also 62,84 statt 64 .

Bernsteinsäure $\mathrm{C}^{8} \mathrm{H}^{8} \mathrm{O}^{8}$. Atom 118 erfordert $14 \mathrm{At}$. $\mathrm{O}$.

$0,117 \mathrm{Grm}$. mit 1,5 CuO gaben 0,10929 O. 1 Atom also 110,2 statt 112 .

$\mathrm{Zu}$ diesen Versuchen reichten Glasröhren von 10 bis 12 Zoll Länge und 2 Linien innerem Durchmesser hin.

Camphor $\mathrm{C}^{20} \mathrm{H}^{16} \mathrm{O}^{2}$. Atom 152 erfordert 54 At. 0 .

$0,102 \mathrm{Grm}$. wurden als kleine Körner mit $6 \mathrm{Grm}$. CuO in der Glasröhre (sie war 2 Fufs lang, 2 Linien im Durchmesser) mit einem Drabte gemengt und noch $\mathbf{8 , 0}$ gekörntes $\mathrm{CuO}$ vorgelegt. Gefunden 0,28991 O. 1 Atom also 432 (gerado 54 At.).

*) Bd. I, Seite 149. 
Benzoèsäure $\mathrm{C}^{14} \mathrm{H}^{8} \mathrm{O}^{4}$. Atom 122 erfordert 30 At. $O$.

0,101 Grm. mit $3 \mathrm{CuO}$ gaben 0,19638 O. 1 At. also 237,2 statt 240 . Zimmtsäure $\mathrm{C}^{18} \mathrm{H}^{8} \mathrm{O}^{4}$. Atom 148 erfordert 40 At. 0 .

0,101 Grm. mit 4,5 CuO gaben 0,21714 O. 1 At. also 318,18 statt 320 . Harnsäure $\mathrm{C}^{10} \mathrm{~N}^{4} \mathrm{H}^{4} \mathrm{O}^{6}$. Atom 168 erfordert 18 At. $\mathrm{O}$.

$0,1015 \mathrm{Grm}$. mit 1,5 $\mathrm{CuO}$ gaben 0,086014 O. 1 Atom also 142,35 statt 144.

Salmiak $\mathrm{NH}^{4} \mathrm{Cl}$. Atom 53,4 erfordert 3 At. $\mathrm{O}$.

0,305 Grm. in Stücken ans Ende der Röhre, davor 3,0 CuO und $1,5 \mathrm{NaO}, \mathrm{CO}^{2}$. Das $\mathrm{CuO}$ war auf etwa $2 / 3$ der Lïnge reducirt. Mit $\mathrm{Fe}^{2} \mathrm{Cl}^{3}$ gab es 0,13494 O. 1 Atom also 23,62 o statt 24 .

Kaliumeisencyanür $\mathrm{C}^{8} \mathrm{~N}^{3} \mathrm{FeK}^{2}+3$ aq. Atom 211,4. 2 Atome erfordern 31 At. $\mathrm{O}$.

0,1 Grm. mit 1,5 CuO - 0,05839 O. 2 At. also 246,8 statt 248 .

Kaliumeisencyanid $\mathrm{C}^{12} \mathrm{~N}^{6} \mathrm{Fe}^{2} \mathrm{~K}^{3}$. Atom 329,6 erfordert 30 A.t. $O$.

0,1015 Grm. mit 1,5 CuO gaben 0,074 O. 1 At. also 240,3 statt 240.

Die Richtigkeit der beim Kaliumeisencyanür und Cyanid gemachten Annahme, dafs das Kupferoxyd das Eisen zu Eisenoxyd $\left(\mathrm{Fe}^{2} \mathrm{O}^{3}\right)$ oxydire, ist freilich nicht erwiesen, aber doch für diese Untersuchungsmethode gültig, da hier aus $2 \mathrm{Fe}$, mögen sie sich zu $\mathrm{Fe}^{2} \mathrm{O}^{3}$ oder zu $2 \mathrm{FeO}$ oxydiren oder ganz unverändert bleiben, beim Auflösen in $\left(\mathrm{Fe}^{2} \mathrm{O}^{3}\right.$, $3 \mathrm{SO}^{3}$ ) stets $6 \mathrm{At}$. $\mathrm{FeO}, \mathrm{SO}^{3}$ hervorgehen, welche $3 \mathrm{At}$. O rom Chamäleon aufnehmen.

1) $2 \mathrm{Fe}+3 \mathrm{CuO}=\mathrm{Fe}^{2} \mathrm{O}^{3}+3 \mathrm{Cu}$. Diese gelöst in : $3 \mathrm{SO}^{3}$ und $2\left(\mathrm{Fe}^{2} \mathrm{O}^{3}, 3 \mathrm{SO}^{8}\right)=3\left(\mathrm{CuO}, \mathrm{SO}^{3}\right)+6\left(\mathrm{FeO}, \mathrm{SO}^{3}\right)$.

2) $2 \mathrm{Fe}+2 \mathrm{CuO}=2 \mathrm{FeO}+2 \mathrm{Cu}$. Diese in : $2 \mathrm{SO}^{3}$ und $2\left(\mathrm{Fe}^{2} \mathrm{O}^{9}, 3 \mathrm{SO}^{3}\right)=2\left(\mathrm{CuO}, \mathrm{SO}^{3}\right)+6\left(\mathrm{FeO}, \mathrm{SO}^{3}\right)$.

3) $2 \mathrm{Fe}+2\left(\mathrm{Fe}^{2} \mathrm{O}^{3}, 3 \mathrm{SO}^{3}\right)=6\left(\mathrm{FeO}, \mathrm{SO}^{3}\right)$.

Schoefelcyankalium C $^{2} \mathrm{NKS}^{2}$. Atom 97,2 erfordert 11 At. 0 .

0,1015 Grm. mit $2 \mathrm{CuO}-0,09107$ O. 1 At. also 87,2 statt 88 .

Da der Schwefel hier vollkommen zu Schwefelsäure $\left(\mathrm{SO}^{3}\right)$ verbrannte, so konnte dieses Verfahren zur Bestimmung des Sauerstoffgehaltes der Polythionsäuren dienen. Ich habe den Versuch gemacht mit dem :

Unterschwefligsaurem Natron $\mathrm{NaO}, \mathrm{S}^{2} \mathrm{O}^{2}+5$ aq. Atom 124,2 erfordert 4 At. 0 .

0,301 Grm. mit 1,5 $\mathrm{CuO}, 0,75 \mathrm{NaO}, \mathrm{CO}^{2}$, davor 2,5 gekörntes $\mathrm{CuO}$. Gefunden 0,075495 O. 1 Atom also 31,20 statt 32 .

Die Schwefelbestimmung würde $2 \mathrm{At}$. $\mathrm{SO}^{3}$ oder $\mathrm{S}^{2} \mathrm{O}^{8}$ liefern, wovon die zur Oxydation nöthig gefundenen 40 abgezogen $\mathrm{S}^{2} \mathrm{O}^{2}$ Iassen. 
Salpetersaurer Harnstoff $\mathrm{C}^{2} \mathrm{H}^{5} \mathrm{~N}^{3} \mathrm{O}^{8}$ oder $\mathrm{C}^{2} \mathrm{H}^{4} \mathrm{~N}^{2} \mathrm{O}^{2}, \mathrm{HO}, \mathrm{NO}$. Atom 123 erfordert 1 At. $O$.

1,235 Grm. mit $1,5 \mathrm{CuO}$ verbrannte ruhig; 0,0927 o. 1 Atom also 9,23 statt 8 .

Da der salpetersaure Harnstoff noch etwas gelblich war, wurde er nochmals sorgfältig gereinigt. Er war nun ganz weifs, gab aber kein besseres Resultat.

1,233 Grm. mit 1,5 CuO gaben 0,0928 O. 1 At. also 9,25 statt 8 .

Bei der trockenen Destillation giebt er nach Pelouze*) Kohlensäure, Ammoniak, Wasser und Stickstoffoxydul. Die Bildung des letzteren hat sich hier also nicht verbindern lassen, jedoch sind von den 5 Atomen $O$ (40) der Salpetersäure nur 1,25 damit entwichen, der Rest ist zu Kohlensäure und Wasser geworden.

Pikrinsaures Kali $\mathrm{C}^{12} \mathrm{~N}^{3} \mathrm{H}^{2} \mathrm{KO}^{14}$ oder $\mathrm{C}^{12}\left(\mathrm{NO}^{4}\right)^{9} \mathrm{H}^{2} \mathrm{KO}^{2}$. Atom 267,2 erfordert 13 At. 0.

$0,1015 \mathrm{Grm}$. mit $3 \mathrm{CuO}$ verbrannte ganz ruhig. Gefunden 0,0462 O. 1 Atom also 121,6 statt 104 .

Von den 12 At. $O(96)$ der 3 At. NO $\mathrm{NO}^{4}$ sind also 17,6 entwichen, wohl als Stickstoffoxyd, 78,4 abor doch zu Wasser und Kohlensäure geworden.

Salpetersüure, organische Basen und Nitroverbindungen eignen sich danach für diese Probe nicht.

So weit reichen meine Versuche. Ich muls es nun dem Urtheil der Chemiker anheimstellen, ob diese Probe für die organische Analyse von Nutzen sein kann, wie ich dieses gehofft habe. Ich glaube, sie würde in den Händen derer, welche mehr Uebung in diesen Arbeiten haben, wie ich, noch wesentlich verbessert werden.

*) Gmelin, Bd. IV, 296.

Hannover, den 23. Augusi 1860. 